\title{
"INFLUENCIA DE LA PERSONALIDAD, LA REGULACIÓN EMOCIONAL, LOS ESTILOS PARENTALES Y ALGUNAS CARACTERÍSTICAS SOCIODEMOGRÁFICAS SOBRE EL ACOSO ESCOLAR EN UN GRUPO DE ADOLESCENTES BRASILEÑOS”
}

\author{
Alejandro J. Mena ${ }^{1}$ \\ Universidad Rafael Landivar, Guatemala \\ Pontificia Universidade Católica do Rio Grande do Sul, Brasil
}

Adriane Arteche

Pontificia Universidade Católica do Rio Grande do Sul, Brasil

\begin{abstract}
RESUMEN
El presente estudio ofrece resultados sobre la asociación entre estilos parentales, personalidad y regulación emocional en los factores de bullying. Participaron 423 adolescentes de dos escuelas públicas en Brasil. Se aplicó un Cuestionario de Datos Sociodemográficos, la escala Exigencia y Responsividad, BFP, Escala de Dificultades de Regulación Emocional (DERS) y el CMIE-III. Se observó que hubo correlación entre el estilo parental negligente y los factores bullying extremo y abusador; entre el factor de personalidad neuroticismo y los factores bullying extremo y víctima. La técnica Manova permitió identificar efecto principal significativo en las variables sexo, grupo étnico, grado de escolaridad de la madre y los estilos parentales. Un análisis de regresión explicó $23 \%$ de la varianza para el factor abusador, manteniendo en el modelo las variables: sexo, escolaridad de la madre, padres viviendo juntos, extroversión, el estilo parental autoritativo y socialización. Para el factor víctima el modelo explicó 18\% de la varianza incluyendo las variables: sexo, edad, abertura, neuroticismo, realización, limitación de estrategias emocionales y poca claridad en objetivos planteados. El estudio amplía las implicaciones de los otros perfiles de bullying asociados a las variables analizadas.
\end{abstract}

\section{Palabras Clave}

Acoso Escolar; Estilos parentales; personalidad; regulación emocional

\begin{abstract}
This study aimed to investigate the association between parenting styles, personality and emotion regulation among bullying factors. The sample consisted of 423 teenagers from southern Brazil. Participants answered a sociodemographic questionnaire, the scale demand and responsiveness, BFP (personality), Emotional Regulation Difficulties Scale (DERS) and the CMIE-III. Results revealed a significant correlation between neglectful parenting style, bullying and extreme bullying. Among neuroticism personality factor, extreme bullying, bullying, passive bystander and victim. Similarly, the factors limited access to emotion regulation strategies and difficulty in controlling impulses were correlated with extreme bullying and victim factors. The analysis of the data by the Manova technique identified significant main effect on the variables sex, ethnicity, education level of mothers and parenting styles. A regression analysis explained $23 \%$ of the variance for the bullying factor, keeping in the model the variables: sex, mother's schooling, parents living together, extroversion, authoritative parental style and socialization. For the victim factor, the model explained $18 \%$ of the variance, including the variables: sex, age, openness, neuroticism, achievement, limitation of emotional strategies and lack of clarity in the objectives set. The study broadens the implications of the other bullying profiles associated with the analyzed variables.
\end{abstract}

Key words

Bullying; parenting styles; emotional regulation; personality

\footnotetext{
Correspondence about this article should be addressed to Alejandro J. Mena.Email: jandromb@hotmail.com; ajmena@url.edu.gt
} 
THE INFLUENCE OF PERSONALITY, EMOTIONAL REGULATION, PARENTING STYLES AND SOME SOCIO-DEMOGRAPHIC VARIABLES ON BULLYING FACTORS AMONG BRAZILIAN TEENAGERS.

Mundialmente $20 \%$ de los niños y jóvenes en edad escolar presentan algún tipo de problemas relacionados con la salud mental (Silva, Oliveira, Mello, Prado, Silva, \& Malta, 2019; WHO, 2005), independientemente del tipo de situación que conduce a una intervención o tratamiento (WHO, 2001). Diversos estudios han demostrado que víctimas del acoso escolar o bullying tienden a desarrollar una amplia gama de dificultades psicológicas como ansiedad, depresión, aislamiento social, problemas de alimentación y diversas patologías de índole somáticas como dolores de cabeza, gastritis, fatiga, ensoñación, etc. (Sansone, Lamc \& Wiederm, 2010). El fenómeno bullying es definido por Olweous (2011) como un comportamiento intencional negativo que busca causar daño en personas o grupos de personas que tienen dificultad para defenderse a sí mismas. Dicho desbalance de poder se repite o tiene el potencial de ser repetido en el tiempo. Además, el fenómeno bullying tiene alcances que transcienden el tiempo y el contexto escolar, considerando que sus consecuencias impactan el bienestar biopsicosocial de quien lo padece (Zhang, De Luca,Oh, Liu, \& Song, 2019; Zhang et al.,2019).

Aunque el fenómeno bullying ha sido comúnmente asociado con el contexto escolar, existen evidencias que indican que es un fenómeno causado por múltiples y complejos motivos, algunos de ellos relacionados con el contexto (grado de cohesión social en la escuela) y otros con características individuales (Boulton \& Smith, 1994; Georgiou, 2008; Springer, Cuevas Jaramillo, Ortiz Gómez, Case, \& Wilkinson, 2016; Wang, Iannotti \& Nansel, 2009). En ese orden de ideas, autores como Ladd (1992) señalan que el contexto familiar ayuda a moldear la capacidad de los niños para lidiar con situaciones del ambiente escolar e impactar en los estilos de relacionamiento social, tanto para manejar adecuadamente experiencias de acoso en la escuela, así como participar de ellas.

A pesar de que el acoso escolar ocurra sin una única causa o motivación y aunque suceda en la mayor parte de los centros de estudio (López \& Saavedra, 2003) es sabido que está vinculado directa o indirectamente al ambiente (Berger, 2007), a las características de personalidad, rasgos familiares (Olweus, 1993,2006,2011) y otras variables que van más allá del ambiente escolar (Leff, Power, Costigan \& Manz, 2003; Elgar et. al., 2013). En este sentido, autores como Sutton y Keogh (2000) señalan que muchos de los estudios realizados vinculados al acoso escolar no han profundizado en variables más extensas y profundas como el constructo personalidad; y cuando lo han hecho, los datos de la literatura no son multidimensionales, presumiblemente por la amplia cantidad de taxonomías existentes para evaluar dichos constructos.

En ese sentido, en el presente estudio el constructo personalidad fue evaluado a la luz de la taxonomía Big Five. Tal clasificación además de ser ampliamente utilizada en diversos estudios cuenta con el crédito de la comunidad científica (Groslin, Rentfrow \& Swann, 2003). De tal manera, el modelo Big Five sostiene que existen cinco factores básicos de personalidad, los cuales se manifiestan repetida y constantemente en la evaluación de los rasgos más comunes de las personas (Cavalcanti \& Pimentel, 2016; John \& Srivastava, 1999), dicho modelo describe el constructo personalidad a través de los factores: Neuroticismo, extroversión, socialización, realización y abertura. De esa manera el factor Neuroticismo indica la tendencia de algunas personas de manifestar malestar psicológico, baja tolerancia a la frustración y respuestas emocionales poco adaptativas. Así mismo, el factor extroversión aparece comúnmente en personas altamente sociables, activas, optimistas y afectuosas. Por su parte, el factor socialización indica que personas con altos resultados en esta área tienden a ser altruistas, generosas, amables y con altos niveles de confianza en las personas. A su vez, el factor realización indica que personas con altas puntuaciones en este componente tienden a ser competentes y altamente comprometidas. Por último, el factor abertura se refiere a personas con alta capacidad imaginativa, curiosas, creativas y abiertas a ideas poco convencionales. 
Aunque las dinámicas e interacciones vinculadas con el acoso escolar despierten de manera previsible sentimientos de frustración y ansiedad tanto en las personas que lo viven como en las personas cercanas al contexto donde eso sucede, es también sabido que esa sensibilidad es marcadamente mayor en algunas personas (Wilton \& Craig, 2000; Frizzo, Bisol \& Lara, 2013). La literatura al respecto sugiere que víctimas de bullying tienden a desarrollar mayores índices de frustración y ansiedad comparado con abusadores, observadores pasivos y observadores activos (Perry, Kusel \& Perry, 1988; Lynn Hawkins, Pepler y Craig, 2001 \& Frizzo, Bisol \& Lara, 2013). De hecho, en los últimos años se han divulgado diversos estudios que informan cómo la depresión y la ansiedad se vinculan con la regulación emocional situaciones potencialmente presentes en los casos de acoso escolar (Andrés, Castañeiras, Canet \& Richaud, 2016).

Cabe mencionar que reconocer tanto las propias como las emociones en otros es un indicador de ajuste psicológico así algunos autores mencionan que las dificultades en el manejo de las emociones tienden a dar como resultado la implementación de estrategias desadaptativas a nivel social (Sanchez, Ortega \& Menesini, 2012; Valle, Betegón, \& Irurtia, 2018). Por lo tanto, considerar el efecto de la regulación emocional puede aportar información que explique con mayor amplitud la dinámica alrededor del acoso escolar. De acuerdo con lo anterior, diversos estudios describen el constructo regulación emocional como la capacidad para integrar y administrar componentes vinculados con las emociones, incluyendo la capacidad de mostrar estados emocionales internos, la orientación de los procesos cognitivos, la autorregulación, el autocontrol y las respuestas conductuales apropiadas para la situación (Espelage, Rose \& Polanin, 2015; Wilton \& Craig, 2000). Es importante resaltar que para el presente estudio el constructo Regulación emocional es analizado desde la perspectiva planteada por Gratz y Roemer (2004) quienes lo analizan desde una perspectiva integrativa y multidimensional que incluye los siguientes factores: a) No aceptación de las emociones negativas, b) incapacidad para establecer objetivos ante la presencia de emociones negativas, c) problemas para controlar el comportamiento impulsivo cuando se experimenta emociones negativas, d) acceso limitado a estrategias de regulación emocional, e) falta de consciencia emocional y por último f) falta de claridad emocional. A pesar de que el acoso escolar cuenta con una amplia gama de publicaciones, pocos estudios resaltan la importancia de elementos de la socialización familiar, por ejemplo, de los estilos parentales. Los estilos parentales se describen como el grado de participación, control y coerción que los padres ejercen sobre sus hijos (Gómez-Ortiz, Del Rey, Romera \& Ortega-Ruiz, 2015; Jiménez-Barbero, Ruiz-Hernández, VelandrinoNicolás, \& Llor-Zaragoza, 2016). Históricamente los tres estilos parentales comúnmente citados en la literatura son el estilo autoritativo, autoritario y permisivo (Baumrind, 1967; Wittig \& Rodriguez, 2019). De esa manera, el estilo autoritativo describe a los padres que muestran una gran capacidad de respuesta, exigencia y autonomía con sus hijos; por su parte un estilo autoritario se refiere a un enfoque en el que los padres se involucran con poca capacidad de respuesta, alta exigencia y bajos niveles de independencia; y un estilo de crianza permisivo se refiere a los padres que expresan altos niveles de capacidad de respuesta y autonomía, pero bajos niveles de exigencia (Darling y Steinberg, 1993; Jiménez-Barbero, Ruiz-Hernández, Velandrino-Nicolás, \& Llor-Zaragoza, 2016; Smetana, 2017).

Respecto de los estilos parentales y el acoso escolar estudios como el de Georgiou (2008) refieren que una alta responsividad (afecto) suele estar asociada con el perfil de víctima, mientras que una moderada responsividad se asocia con la empatía y el buen trato hacia quienes son percibidos como débiles. Dicho estudio postula que la línea entre los efectos de una alta y moderada responsividad es tenue. Para el presente estudio el constructo estilos parentales se plantea desde el modelo teórico propuesto por Costa y Texeira (2000) quienes con base en el trabajo de Braumind (1967) y Maccoby y Martin (1983) proponen cuatro estilos parentales que surgen de la combinación de las dimensiones, exigencia (control) y responsividad (afecto), a saber: autoritativo, autoritario, 
indulgente y negligente. De esa forma el fenómeno bullying se presenta de manera multidimensional y en diversos contextos, es por ello que debe abordarse desde un contexto familiar, desde la dinámica de las instituciones y desde las particulares causas y efectos en las personas (Millan, Barrera \& Ospina, 2015; Olson, Lopez-Duran, Lunkenheimer, Chang \& Sameroff, 2011). En ese sentido, es importante resaltar que, aunque la literatura respecto del tema muestra una marcada expansión, se requiere de evidencias que aporten mayores respuestas respecto del rol que ejercen variables como la personalidad, la regulación emocional, los estilos parentales e información sociodemográfica en el acoso escolar. Tradicionalmente y desde los estudios iniciales realizados por Olweous en la década de los setenta, muchos de los estudios que abordan el fenómeno del acoso escolar, parten principalmente de perspectivas bidimensionales en el análisis del fenómeno, es decir analizando casi exclusivamente al acosador y a la víctima (Olweous, 2016). Sin embargo, estudios más recientes analizan de manera más extensa a quienes participan directa o tácitamente en los actos de acoso. De esa manera el modelo postulado por Caballo, Calderón, Arias, Salazar y Irurtia (2012) permite identificar los actos de acoso desde los distintos roles o perfiles en los que potencialmente pueden participar los niños y jóvenes, siendo estos: acosador extremo y ciberacosador, acosador, observador activo, observador pasivo y víctima.

Es importante reiterar que a pesar de la expansión la literatura respecto del acoso escolar se percibe un evidente vacío de estudios que vinculen la existencia del acoso con aspectos que van más allá del contexto escolar. Como consecuencia de ello, el presente estudio pretende: (1) investigar si existe relación entre personalidad, regulación emocional y estilos parentales en cada uno de los factores asociados al acoso escolar (acoso extremo, abusador, observador pasivo, observador activo y victima) y (2) Identificar de qué manera las variables analizadas (Personalidad, regulación emocional, estilos parentales y variables sociodemográficas) expresan de manera conjunta la frecuencia de sucesos de acoso según cada uno de los factores.

En resumen, se busca presentar una explicación que posibilite la integración de una perspectiva amplia para la comprensión de los procesos ligados al acoso escolar a través de la descripción de factores potencialmente asociados. Concretamente y como resultado de lo expuesto, se formula la hipótesis que las variables personalidad, regulación emocional y estilos parentales están asociadas a los factores del acoso escolar. Se pretende que los hallazgos sean de utilidad en la toma de decisiones para diversas instancias que trabajen con la problemática del acoso escolar.

\section{Metodología}

El presente estudio parte de una perspectiva descriptiva, cuantitativa, transversal y correlacional.

\section{Participantes}

Se utilizó un muestreo no probabilístico por conveniencia, donde participaron voluntariamente (posterior a la firma del término de consentimiento informado tanto de los padres como de los adolescentes) 423 adolescentes inscritos regularmente en 1ro, 2do, y 3er grado de enseñanza media de dos escuelas secundarias de Porto Alegre, Rio Grande do Sul, Brasil. Cada una de las escuelas albergaba aproximadamente a 300 estudiantes de secundaria al momento de realizar la investigación. Del total de participantes $57 \%$ eran mujeres y $43 \%$ hombres, y la edad de los y las participantes estuvo comprendida entre 14 y 18 años $(\mathrm{M}=16.3$; DS=.99).

Conforme a las peticiones de ambos centros de estudio, la recolección de los datos se realizó en las respectivas salas de aula en dos momentos diferentes para cada grupo. El tiempo total para responder las escalas fue de aproximadamente 45 minutos. 


\section{Instrumentos}

Se aplicó el Cuestionario Multimodal de Interacción Escolar CMIE-III (Caballo et al., 2012). La escala cuenta con 46 preguntas de opción múltiple en formato Likert indicando cuantas veces el participante vivió eventos de bullying o acoso escolar. Los y las respondientes deben indicar cuanto experimentaron cada situación a partir del inicio del presente ciclo escolar, de la siguiente manera: (1) nunca, (2) Pocas veces, (3) bastantes veces y (4) muchas veces, los valores iban desde 1 punto para nunca hasta 4 puntos para muchas veces. El instrumento brinda resultados para cinco factores asociados al acoso escolar o bullying: Bullying extremo, abusador, observador activo, observador pasivo y víctima. En la presente investigación se determinó la confiabilidad de los puntajes mediante el Alfa de Cronbach, obteniéndose un valor de .87, que lo sitúa con un alto nivel de consistencia interna.

Escala de Estilos Parentales Responsividad y Exigencia (Costa, Texeira \& Gomes, 2000). La escala está compuesta por seis preguntas sobre la percepción de los adolescentes acerca del grado de exigencia de los padres y diez preguntas acerca del grado de responsividad. La escala está confeccionada en formato Likert de 1 a 3 puntos, de esa manera, cuanto más cerca de tres, mayor nivel de exigencia o responsividad percibida por los participantes. La combinación de los resultados altos y bajos de las dos escalas permite clasificar cuatro tipos de estilos parentales: Autoritario (alta exigencia y baja responsividad), autoritativo (alta exigencia y alta responsividad), indulgente (baja exigencia y alta responsividad) y negligente (baja responsividad y baja exigencia). La versión brasileña de la escala mostró buenos niveles de consistencia interna: $\alpha=.72$ y $\alpha=.78$.

Batería Factorial de Personalidad (Nunes, Hutz \& Nunes, 2010). Este instrumento está basado en la escala Big Five de Costa y McCrae (1992) y está compuesto por 126 preguntas que abarcan los factores de personalidad Neuroticismo, Extroversión, Socialización, Realización y Abertura. Las respuestas son registradas en una escala Likert que va desde 1 hasta 7 puntos de acuerdo con el grado de identificación de la persona evaluada con cada ítem. El análisis factorial y de consistencia interna arrojaron la presencia de cinco factores principales, en sintonía con el modelo original. Las escalas mostraron índices de consistencia interna de entre $\alpha=.74$ para el factor Abertura y $\alpha=.89$ para el factor Neuroticismo (Nunes, Hutz \& Nunes, 2010).

Escala de Dificultades de Regulación Emocional (DERS) elaborada por Gratz e Roemer (2004). La escala evalúa elementos característicos de la regulación emocional en seis niveles: No aceptación de las emociones negativas, incapacidad para establecer objetivos ante la presencia de emociones negativas, problemas para controlar el comportamiento impulsivo cuando se experimenta emociones negativas, acceso limitado a estrategias de regulación emocional, falta de consciencia emocional y, por último, falta de claridad emocional. La escala consta de 36 items en formato Likert que va de 0 a 4 puntos de la siguiente manera: $0=$ nunca, $1=$ pocas veces, $2=$ a veces, $3=$ muchas veces $y$ $4=$ siempre. El análisis de consistencia interna para la versión brasilera arrojó un índice de alpha de Cronbach de .93 (Coutinho, Ribeiro, Ferreirinha \& Dias, 2009).

\section{Procedimiento}

Para la realización del presente estudio se procedió a establecer contacto con dos escuelas de enseñanza media en Porto Alegre, Brasil. El investigador se desplazó a dichas escuelas para detallar informaciones acerca de la investigación. El protocolo de consentimiento informado fue enviado a padres y cuidadores y recolectado a través del departamento de Orientación Escolar de cada una de las escuelas. Luego de la obtención de la autorización por parte de los padres y cuidadores se procedió a entregar un término de consentimiento para los adolescentes con información acerca del estudio. Los alumnos completaron las pruebas aplicadas en el salón de clase y en horarios pactados con las 
direcciones de cada centro educativo, necesitando aproximadamente una hora para completar dicha tarea.

\section{Análisis estadísticos}

Para el análisis de los resultados fue utilizado el paquete estadístico SPSS versión 23.0. Inicialmente fueron realizados análisis descriptivos (distribución, media y desviación estándar) y de consistencia interna de los instrumentos utilizados. Posteriormente se procedió a realizar series de MANOVAS con el fin de investigar el efecto de las variables sociodemográficas sobre los factores de bullying o acoso escolar (Bullying extremo, abusador, observador activo, observador pasivo y víctima) Así como para investigar el efecto de los estilos parentales. Para el análisis de los estilos parentales se procedió a determinar cuatro estilos parentales a partir de las escalas de exigencia y responsividad, tal como lo sugieren sus autores. De esa manera, fue utilizada la mediana como punto de corte para clasificar cada nivel alto/bajo (exigencia=9; responsividad=14). Posteriormente fue realizada una correlación de Pearson entre las categorías de bullying y los resultados en las sub-escalas de personalidad y regulación emocional. Por último, con los datos proporcionados por los análisis multivariados y las correlaciónales para la condición de acoso escolar, se elaboró cinco series de regresiones lineares teniendo como variable dependiente cada una de las categorías de acoso escolar (bullying extremo, abusador, observador activo, observador pasivo y victima) y como variables independientes los factores significativamente asociados a cada categoría $(\mathrm{p}<.05)$ de personalidad, regulación emocional, estilos parentales y datos sociodoemográficos.

\section{Resultados}

Pudo observarse un bajo porcentaje de missings en los instrumentos aplicados, variando de $.5 \%$ (Estilos Parentales) y 3.42\% (CMIE-III). Para fines de los análisis, los datos faltantes fueron introducidos a través de la media de serie. Además como se observa en la tabla 1 se trata de un grupo de estudiantes conformado principalmente por mujeres $(57 \%)$, quienes además reportaron pertenecer principalmente a la raza blanca $(56.8 \%)$, afro descendiente $(32.70 \%)$ y mestiza $10.60 \%)$. 
Tabla 1

Frecuencia y porcentaje de las variables sociodemográficas

\begin{tabular}{|c|c|c|c|}
\hline & & $F(n=423)$ & $\%$ \\
\hline \multirow[t]{2}{*}{ Sexo } & Femenino & 241 & 57.00 \\
\hline & Masculino & 182 & 43.00 \\
\hline \multirow[t]{5}{*}{ Edad } & 14 & 13 & 3.10 \\
\hline & 15 & 105 & 24.80 \\
\hline & 16 & 153 & 36.20 \\
\hline & 17 & 116 & 27.40 \\
\hline & 18 & 36 & 8.50 \\
\hline \multirow[t]{3}{*}{ Etnia } & $\begin{array}{l}\text { Afrodescendient } \\
\mathrm{e}\end{array}$ & 133 & 32.70 \\
\hline & Blanca & 231 & 56.80 \\
\hline & Parda (Mestiza) & 43 & 10.60 \\
\hline \multirow{3}{*}{$\begin{array}{l}\text { Repitencia } \\
\text { s Escolar }\end{array}$} & No repetido & 212 & 50.70 \\
\hline & Repitió una vez & 128 & 30.60 \\
\hline & $\begin{array}{l}\text { Repitió Dos o } \\
\text { más grados }\end{array}$ & 78 & 18.70 \\
\hline \multirow{2}{*}{$\begin{array}{l}\text { Padres } \\
\text { viviendo } \\
\text { juntos }\end{array}$} & $\mathrm{Si}$ & 191 & 45.40 \\
\hline & No & 230 & 54.60 \\
\hline \multirow{3}{*}{$\begin{array}{l}\text { Nivel de } \\
\text { educación } \\
\text { de la } \\
\text { madre }\end{array}$} & Primaria & 154 & 36.40 \\
\hline & $\begin{array}{l}\text { Enseñanza } \\
\text { media }\end{array}$ & 182 & 43.00 \\
\hline & $\begin{array}{l}\text { Superior } \quad y / o \\
\text { posgrado }\end{array}$ & 60 & 14.20 \\
\hline \multirow{3}{*}{$\begin{array}{l}\text { Nivel de } \\
\text { educación } \\
\text { del padre }\end{array}$} & primaria & 158 & 37.40 \\
\hline & $\begin{array}{l}\text { Enseñanza } \\
\text { media }\end{array}$ & 150 & 35.50 \\
\hline & $\begin{array}{l}\text { Superior } \quad y / o \\
\text { posgrado }\end{array}$ & 60 & 14.20 \\
\hline
\end{tabular}

Posteriormente se analizó el efecto de las variables demográficas en los factores del acoso escolar. De esa manera, el MANOVA mostró un efecto significativo de la variable sexo $(\mathrm{F}(5.417)=11.21, \mathrm{p}=.01)$. Además, como puede observarse en la tabla 2 la inspección de los ANOVAS reveló que los chicos obtuvieron resultados significativamente mayores que las chicas en los factores abusador [chicos $\mathrm{M}=17.969(\mathrm{DT}=5.74)$; chicas $\mathrm{M}=14.81(\mathrm{DT}=4.24)$ ] y víctima [chicos $\mathrm{M}=12.19(\mathrm{DT}=4.21)$; chicas $\mathrm{M}=11.35(\mathrm{DT}=3.68)$ ]. Respecto de la variable edad, el MANOVA no mostró efecto principal significativo [F (10. $834)=1.57, \mathrm{p}=.11 \mathrm{]}$. Se observaron diferencias significativas en el factor víctima y en el factor bullying extremo. Análisis post hoc (LSD) indicaron que en el factor bullying extremo los adolescentes en la franja de edad de 16 años reportaron promedios significativamente más altos que jóvenes en la franja de los 17-18 años [16 años $\mathrm{M}=10.35(\mathrm{DT}=2.91) ; 17-18$ años $\mathrm{M}=9.75(\mathrm{DT}=2.21)$; $\mathrm{p}<.05]$; por su parte para el factor victima nuevamente el grupo en la fase de los 16 años reportó promedios 
significativamente mayores que jóvenes entre 14-15 años [14-15 años $\mathrm{M}=11.27(\mathrm{DT}=3.11) ; 16$ años $\mathrm{M}=12.48(\mathrm{DT}=2.48) ; \mathrm{p}<.05]$.

La variable grupo étnico mostró un efecto principal significativo según los análisis multivariados del MANOVA $[\mathrm{F}(10.802)=2.01 ; \mathrm{p}=.03]$. Debido al hecho de no haber sido observados efectos significativos y en función del efecto principal observado se realizó una serie análisis post hoc. De esa manera, los resultados revelaron diferencias significativas en el factor abusador cuando se comparó el grupo identificado como perteneciente a la raza negra y el grupo identificado como raza blanca [blancos $\mathrm{M}=15.80(\mathrm{DT}=5.24)$, raza negra $\mathrm{M}=17.01(\mathrm{DT}=5.07) ; \mathrm{p}>.05)]$. De la misma manera, en el factor observador activo el grupo perteneciente a la etnia blanca presentó diferencias significativas comparado con el grupo perteneciente a la etnia negra [blancos $\mathrm{M}=12.13(\mathrm{DT}=3.84)$, negros $\mathrm{M}=11.29(\mathrm{DT}=3.45) ; \mathrm{p}<.05)]$. De la misma manera, en el factor víctima pudo observarse diferencias significativas entre ambos grupos étnicos [blancos $\mathrm{M}=12.06(\mathrm{DT}=4.34)$, negros $\mathrm{M}=11.16(\mathrm{DT}=3.13) ; \mathrm{p}<.05)]$.

Se constató la presencia de diferencias significativas en el factor abusador [padres viviendo juntos $\mathrm{M}=15.31$ (DT=4.69), padres separados $\mathrm{M}=16.91(\mathrm{DT}=4.47)]$.

Por su parte el factor grado de instrucción de la madre indicó que existe efecto estadísticamente significativo en el MANOVA $[\mathrm{F}(10.78)=2.36, \mathrm{p}=.01]$. Posteriormente los ANOVAS expresaron diferencias significativas en el factor abusador. De esa manera los análisis post hoc (LSD) indicaron que las diferencias eran significativas en el grupo de hijos de madres con primaria incompleta $(\mathrm{M}=14.83$, DT $=4.57)$ en comparación con los hijos de madres con enseñanza media completa $(\mathrm{M}=16.98$, DT=5.31; $\mathrm{p}<.01)$.

Finalmente, los resultados del MANOVA no señalaron efectos significativos globales en la variable grado de instrucción del padre [F (10.724)=1.71; p=.08]. Los resultados evidenciaron además efecto significativo en el factor abusador. Los análisis post hoc señalaron que había diferencias significativas en el factor abusador cuando se comparó el nivel de enseñanza del padre; en ese sentido la mayor diferencia se observó cuando el padre tenía primaria completa $(\mathrm{M}=15.27 ; \mathrm{DT}=4,83)$ versus el grupo de padres con instrucción superior o posgrado $(\mathrm{M}=17.68 ; \mathrm{DT}=5.79 ; \mathrm{p}<.01)$. La Tabla 2 resume el efecto de las variables sociodemográficas en los factores evaluados. (TABLA 2)

\section{Efecto de los Estilos Parentales en la condición de Acoso escolar}

De la misma manera se investigó el efecto de los estilos parentales en los factores asociados al acoso escolar. El MANOVA señaló efecto principal significativo en función de los estilos parentales $[\mathrm{F}(15.1251)=2.69 ; \mathrm{p}=.01]$. Dichas diferencias fueron observadas en el factor abusador $[\mathrm{F}(3.419)=7.39 ; \mathrm{p}=.01]$ y en el factor bullying extremo $[\mathrm{F}(3.419)=2.77 ; \mathrm{p}=.04]$, sin embargo no se observó efectos significativos en los factores observador activo $[\mathrm{F}(3.419)=2.55 ; \mathrm{p}=.06]$, observador pasivo $\mathrm{F}(3.419)=1.01 ; \mathrm{p}=.39]$ y víctima $\mathrm{F}(3.419)=1.33 ; \mathrm{p}=.26]$. Los análisis post hoc (LSD) señalaron diferencias significativas en el factor abusador cuando se comparó el estilo parental negligente $(\mathrm{M}=17.70, \mathrm{DT}=5.56)$ con el estilo parental autoritario $(\mathrm{M}=15.97, \mathrm{DT}=4.62 ; \mathrm{p}<.05) ; \mathrm{y}$ cuando se comparó el estilo autoritativo $(\mathrm{M}=14.89, \mathrm{DT}=4.35)$ versus el estilo indulgente $(\mathrm{M}=16.85 ; \mathrm{DT}=6.21 ; \mathrm{p}<.01)$ y versus el estilo negligente $(\mathrm{M}=17.70 ; \mathrm{DT}=5.56 ; \mathrm{p}<.01)$. Por su parte en el factor bullying extremo se identificó diferencias cuando se comparó el estilo autoritativo $(\mathrm{M}=9.71$, DT=2.16) con el estilo negligente $(\mathrm{M}=10.59$; DT=2.93; $\mathrm{p}<.01)$.

\section{Relación entre personalidad, regulación emocional y la condición de acoso escolar}

Entre los resultados más relevantes se subraya que altos resultados vistos en el factor abusador fueron significativamente asociados con mayor extroversión ( $\mathrm{r}=.19$; $\mathrm{p}=<.01)$ y negativamente asociado al factor socialización $(\mathrm{r}=-.29 ; \mathrm{p}=<.01)$. Respecto del factor bullying extremo, éste fue significativamente asociado con resultados más altos en la dimensión de personalidad neuroticismo $(\mathrm{r}=.24 ; \mathrm{p}=<.01)$ y a una gama de dificultades 
vinculadas a la regulación emocional como: limitación de estrategias emocionales ( $\mathrm{r}=.29$; $\mathrm{p}<.01)$, no aceptación de respuestas emocionales $(\mathrm{r}=.18 ; \mathrm{p}<.01)$ dificultad para controlar los impulsos $(\mathrm{r}=.26 ; \mathrm{p}<.01)$, dificultad de actuar de acuerdo con objetivos planteados $(\mathrm{r}=.20 ; \mathrm{p}<.01)$ y falta de claridad emocional $(\mathrm{r}=.21 ; \mathrm{p}<.01)$.

Por su parte, el factor observador activo fue significativamente asociado con altos niveles en la dimensión de personalidad socialización $(\mathrm{r}=.17 ; \mathrm{p}<.01)$. Además, dicho factor fue negativamente asociado a la dimensión de regulación emocional falta de conciencia emocional $(\mathrm{r}=-.13 ; \mathrm{p}<.05)$. El perfil observador pasivo fue asociado con la dimensión falta de claridad emocional $(\mathrm{r}=.18 ; \mathrm{p}<.01)$. Finalmente, para el perfil víctima se observó resultados asociados con la dimensión de personalidad neuroticismo $(r=.26 ; \mathrm{p}<.01)$ y con los factores de regulación emocional: limitación de estrategias emocionales $(\mathrm{r}=.32 ; \mathrm{p}<.01)$, no aceptación de las respuestas emocionales $(\mathrm{r}=.20 ;<.01)$, dificultad en el control de impulsos $(\mathrm{r}=.22 ; \mathrm{p}<.01)$ y dificultad para actuar de acuerdo con objetivos $(\mathrm{r}=.22 ; \mathrm{p}<.01)$.

La media general, para la variable estilos parentales según tipo de participación en los casos de acoso escolar o bullying se presentan a continuación:

Figura 1. Media de estilos parentales según el perfil de participación en actos de acoso escolar.

\section{Media de Estilos Parentales}

20

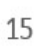

10

5
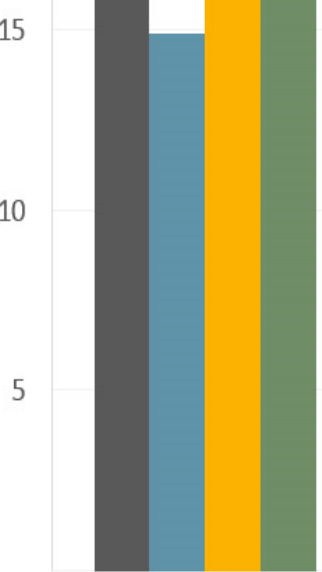

Abusador

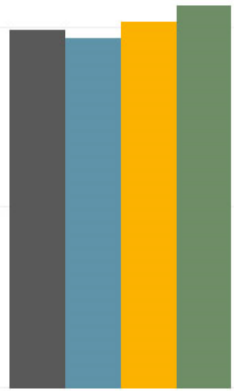

Bullying Extremo

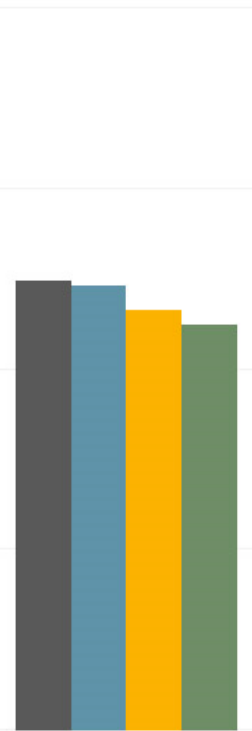

Obs. Activo

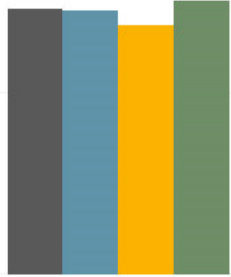

Obs. Pasivo
- Autoritário

- Autoritativo

- Indulgente

- Negligente

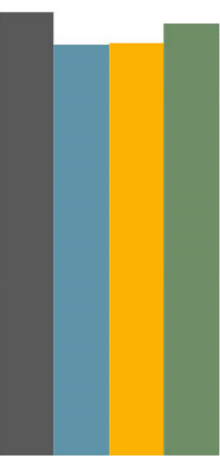

Víctima

Se presentan a continuación correlaciones entre cada uno de los factores de personalidad y regulación emocional para cada uno de los perfiles de bullying: 
Tabla 2

Correlación entre el bullying y las variables personalidad y regulación emocional

\begin{tabular}{|c|c|c|c|c|c|c|}
\hline & & $\begin{array}{l}\text { Bullying } \\
\text { Extremo } \\
\end{array}$ & Abusador & $\begin{array}{c}\text { Observador } \\
\text { Activo }\end{array}$ & $\begin{array}{c}\text { Observador } \\
\text { Pasivo } \\
\end{array}$ & Víctima \\
\hline \multirow{5}{*}{ Personalidad } & Abertura & 0.04 & 0.04 & $.11 *$ & -0.18 & $.09 *$ \\
\hline & Neuroticismo & $.24 * *$ & 0.04 & -0.03 & $0.12 *$ & $.26^{* *}$ \\
\hline & Realización & 0.07 & -0.09 & $.09 *$ & 0.04 & $.11 *$ \\
\hline & Extroversión &, 05 & $.19 * *$ & $.11^{*}$ & -0.08 & -0.03 \\
\hline & Socialización & $-.13 *$ & $-.29 * *$ & $.17 * *$ & -0.01 & -0.06 \\
\hline \multirow{7}{*}{$\begin{array}{l}\text { Regulación } \\
\text { emocional }\end{array}$} & $\begin{array}{l}\text { Regulación } \\
\text { Total }\end{array}$ & 0.29 & 0.03 & 0.08 & $.12^{*}$ & $.32 * *$ \\
\hline & $\begin{array}{l}\text { Limitación } \\
\text { de estrategias }\end{array}$ & $.29 * *$ & -0.01 & 0.07 & $.11^{*}$ & $.32 * *$ \\
\hline & $\begin{array}{l}\text { No } \\
\text { aceptación } \\
\text { de respuestas } \\
\text { emocionales }\end{array}$ & $.18 * *$ & -0.02 & $.12 *$ & 0.02 & $.26^{* *}$ \\
\hline & $\begin{array}{l}\text { Falta de } \\
\text { consciencia } \\
\text { emocional }\end{array}$ & -0.03 & $.11^{*}$ & $-.13 * *$ & -0.01 & -0.04 \\
\hline & $\begin{array}{l}\text { Dificultad } \\
\text { em el control } \\
\text { de impulsos }\end{array}$ & $.26 * *$ & $.11^{*}$ & 0.09 & 0.01 & $.22 * *$ \\
\hline & $\begin{array}{l}\text { Dificultades } \\
\text { respecto de } \\
\text { los objetivos }\end{array}$ & $.20 * *$ & -0.05 & 0.08 & 0.08 & $.25^{* *}$ \\
\hline & $\begin{array}{l}\text { Falta de } \\
\text { claridad } \\
\text { emocional }\end{array}$ & $.21 * *$ & 0.01 & 0.04 & $.18 * *$ & $.21^{*}$ \\
\hline
\end{tabular}

* Correlación significativa para $p<.05 * *$ Correlación significativa para $p<.01$

\section{Modelo de predicción para la condición de acoso en la escuela}

Para identificar la contribución conjunta de las variables independientes asociadas a los factores de acoso escolar se elaboró un análisis de regresión linear múltiple stepwise. De esa manera, para la condición de bullying extremo el modelo presentado fue significativo y responsable por $12 \%$ de la variancia explicada. Las variables que se mantuvieron en el modelo y que mostraron mayor peso fueron: acceso limitado a las estrategias de regulación emocional, así como el estilo parental negligente. Para la condición abusador el modelo mostrado por el análisis de regresión fue significativo y explicó $23 \%$ de la variancia. Las variables mantenidas en el modelo fueron sexo, grado de instrucción de la madre, padres viviendo juntos, extroversión, socialización y el estilo parental autoritativo (negativamente asociado). Cabe destacar el peso del hecho de ser hombre como factor asociado a la ocurrencia de casos de acoso, seguido de la dimensión de personalidad conocida como extroversión. Es importante mencionar que tanto el estilo parental autoritativo como la dimensión de personalidad socialización fueron negativamente asociados con dicho factor. 
El ser hombre se vio asociado al perfil abusador, al igual que el rasgo de personalidad extroversión. De similar manera, el estilo parental autoritativo y el factor de personalidad socialización parecen estar negativamente asociados a ese perfil.

Por su parte, para la condición observador pasivo las variables explicativas: factores de personalidad socialización y abertura en conjunto con el estilo parental negligente (negativamente asociado) y la no aceptación de respuestas emocionales responden por 5\% de la variancia explicada.

Finalmente, en el modelo creado para el perfil denominado observador pasivo, el factor de personalidad neuroticismo y la falta de claridad emocional explicaron 3\% de la variancia. En ese sentido, la variable falta de claridad emocional presentó mayor influencia dentro del modelo. Finalmente, para el factor víctima las variables sexo, edad, abertura, neuroticismo, realización, limitación de estrategias emocionales y limitaciones respecto de la claridad para actuar conforme a objetivos planteados fueron en conjunto responsables por $18 \%$ de la variancia. 
Tabla 4

Modelo de regresión para los factores asociados con el bullying

\begin{tabular}{|c|c|c|c|c|c|c|c|c|}
\hline & Modelo & $\boldsymbol{\beta}$ & $\mathbf{t}$ & $\mathbf{p}$ & $\mathbf{F}$ & G1 & $\mathbf{p}$ & $\mathbf{R}^{2}$ \\
\hline Bullying & (constante) & & & & 12.566 & 5.412 & .01 & .12 \\
\hline \multirow[t]{5}{*}{ Extremo } & Negligente & .12(.17-.24) & 2.60 & .01 & & & & \\
\hline & Neuroticismo & . $.11(.03-.67)$ & 2.15 & .03 & & & & \\
\hline & Realización & . $11(.07-.70)$ & 2.42 & .02 & & & & \\
\hline & Limite Estrategias & $.20(.28-.92)$ & 3.65 & .01 & & & & \\
\hline & Falta de Claridad & $.10(.02-.70)$ & 2.06 & .04 & & & & \\
\hline \multirow[t]{7}{*}{ Abusador } & (constante) & & & & 18.45 & 6.352 & .01 & .23 \\
\hline & Sexo & $.31(2,21-, 19)$ & 6.92 & .01 & & & & \\
\hline & $\begin{array}{l}\text { Escolaridad } \\
\text { Madre }\end{array}$ & $.12(0,21-, 58)$ & 2.59 & .01 & & & & \\
\hline & $\begin{array}{l}\text { Padres viviendo } \\
\text { juntos }\end{array}$ & $.12(0,31-, 21)$ & 2.61 & .01 & & & & \\
\hline & Autoritativo & $-.10[-2.11-(-) .02]$ & -2.00 & .05 & & & & \\
\hline & Extroversión & $.23(.87-2.05)$ & 4.89 & .01 & & & & \\
\hline & Socialización & $-.18[-2.04-(-) .57]$ & -3.49 & .01 & & & & \\
\hline \multirow{5}{*}{$\begin{array}{l}\text { Observador } \\
\text { Activo }\end{array}$} & (constante) & & & & 6.77 & 4.412 & .01 & .05 \\
\hline & Negligente & $-.08(-1.49-.16)$ & -1.58 & .12 & & & & \\
\hline & Socialización & $.15(.31-1.38)$ & 3.09 & .01 & & & & \\
\hline & Abertura & $.12(.13-1.28)$ & 2.42 & .02 & & & & \\
\hline & $\begin{array}{l}\text { No aceptación de } \\
\text { respuestas } \\
\text { emocionales }\end{array}$ & $.10(.03-.84)$ & 2.12 & .03 & & & & \\
\hline \multirow{3}{*}{$\begin{array}{l}\text { Observador } \\
\text { Pasivo }\end{array}$} & (constante) & & & & 7.88 & 2.420 & .01 & .03 \\
\hline & Neuroticismo & $.06(-.11-.46)$ & 1.21 & .23 & & & & \\
\hline & Falta de Claridad & $.16(.20-.83)$ & 3.20 & .01 & & & & \\
\hline \multirow[t]{6}{*}{ Víctima } & (constante) & & & & 18.99 & 5.412 & .01 & .18 \\
\hline & Sexo & $.21(.94-2.38)$ & 4.54 & .01 & & & & \\
\hline & Neuroticismo & $.17(.34-1.29)$ & 3.37 & .01 & & & & \\
\hline & Realización & $.15(.32-1.23)$ & 3.33 & .01 & & & & \\
\hline & Limite Estrategias & $.21(.47-1.50)$ & 3.79 & .01 & & & & \\
\hline & Dific. Objetivos & $.14(.14-1.03)$ & 2.61 & .01 & & & & \\
\hline
\end{tabular}

\section{Discusión}

El presente estudio tuvo como principal objetivo investigar asociación y valor predictivo de la personalidad, la regulación emocional y los estilos parentales, en las diferentes categorías vinculadas al acoso escolar (bullying extremo, abusador, observador activo, observador pasivo y víctima) en una muestra de adolescentes brasileños. Los resultados ofrecen evidencia del peso que tienen el sexo en los casos de acoso, ya que mujeres obtuvieron menores índices de participación en actos de agresión hacia sus pares cuando se compara con los chicos; en ese sentido, la literatura apunta que es culturalmente esperado que las chicas sean más obedientes, sumisas, amables, tolerantes y menos agresivas que los hombres (Binsfeld \& Lisboa, 2010). Además, los resultados indican que 
los hombres sufren más acoso que las mujeres. Debido a lo anterior, es importante resaltar que la mayoría de los instrumentos que evalúan acoso escolar miden principalmente manifestaciones de agresividad predominantemente masculinas o culturalmente esperadas en hombres; por lo que se percibe un vacío de tanto de instrumentos como de modelos teóricos que puedan proveer de información respecto de la agresividad en mujeres, cuyo comportamiento agresivo suele manifestarse sutilmente.

Un hallazgo particular y digno de resaltar en el presente estudio, está relacionado con la interacción que se da entre los diferentes grupos étnicos respecto del acoso en la escuela. Los resultados en el presente estudio indican que participantes que reportaron ser de raza negra mostraron mayor prevalencia en el factor abusador, mientras que quienes reportaron pertenecer a la raza blanca mostraron mayor prevalencia en el factor víctima y observador activo. Tales resultados confirman algunas investigaciones llevadas a cabo en los Estados Unidos de América, en las cuales los participantes de los grupos étnicos mayoritarios muestran más alta prevalencia en el factor víctima y menores índices de participación de acoso en la escuela cuando fueron comparados con miembros pertenecientes a minorías étnicas, particularmente de raza negra (Wang, Iannotti \& Nansel, 2009). Para la presente investigación, una potencial explicación podría ser el hecho de encontrar diferencias culturales en la percepción de agresividad, tomando en consideración que algunas conductas agresivas pueden ser consideradas adaptativas en algunos contextos.

En el presente estudio la principal hipótesis apunta hacia una asociación significativa entre estilos parentales y las categorías vinculadas al acoso en la escuela, tomando en cuenta que la literatura al respecto apunta que las categorías bullying extremo y abusador se encuentran fuertemente ligadas al estilo parental autoritario, mientras que la variable víctima es comúnmente asociada a estilos parentales indulgente y negligente (Baldry \& Farrington, 2000; Garnefski, \& Okma, 1996; Jiménez-Barbero, Ruiz-Hernández, Velandrino-Nicolás, \& Llor-Zaragoza, 2016). En ese sentido el presente estudio difiere de dichos estudios tomando en cuenta que fue el estilo parental negligente el que mostró mayor asociación con las categorías acosador, bullying extremo y observador activo. Debe destacarse que dicha diferencia puede ser explicada a la luz del contexto de los participantes del estudio, considerando que una de las características del contexto familiar de los y las participantes era la ausencia de alguno de los padres. Otro dato interesante es el hecho de que el estilo parental negligente aparece asociado con jóvenes que presentaron perfil de acosadores y de acoso extremo. Lo anterior coincide con el estudio realizado en Australia por Ahmed y Braithwaite (2004), el cual indicó que el porcentaje de jóvenes con padres con estilo negligente es mayor en los adolescentes que participaron en actos de acoso escolar y menor en los adolescentes que no participaron.

Respecto de la dimensión personalidad y su interacción en el proceso de acoso escolar, la muestrea del presente estudio se mostró consistente con lo observado en otras publicaciones internacionales, principalmente respecto del factor neuroticismo. Los resultados del presente estudio muestran que dicho factor se encuentra relacionado con el factor observador pasivo, pero principalmente con el factor bullying extremo y víctima. Esos resultados se encuentran en concordancia con el estudio elaborado por Tani, Greenman, Schneider y Fregoso (2003) quienes señalan que las victimas tienden a mostrar mayor inestabilidad emocional y son más inclinadas a proteger únicamente sus propios derechos. Los hallazgos confirman la importancia de conocer no sólo las características individuales de la víctima, sino como esta interactúa socialmente, considerando que dicho papel suele ser el resultado de las relaciones que este establece con su contexto cercano (Cuervo, Murrieta, \& Martínez, 2018). Además, pudo observarse que el factor extroversión se encuentra significativamente asociado al perfil abusador, tal resultado era esperado tomando en cuenta que los abusadores muestran características vinculadas con la alta expresividad de emociones, sentimientos e ideas así como un alto grado de interés en obtener éxito social (Craig \& Pepler, 1997) y muchas veces muestran poca abertura y alto 
grado de energía o extroversión (Caravita, Di Blasio \& Salmivalli 2010; Garner \& Hinton 2010; Tani, Greenman, Schneider \& Fregoso, 2003). Tales características, presentes en el abusador, suelen ser explicadas por la importancia que estos suelen dar a la aceptación y el estatus social (Balakrishnan, Khan, Fernandez, \& Arabnia, 2019). Con relación a la variable regulación emocional, pudo observarse una alta asociación principalmente entre sus componentes y los factores asociados al acoso en la escuela denominados bullying extremo y víctima, los cuales mostraron alta asociación con los sub-factores limitación de estrategias emocionales, no aceptación de las respuestas emocionales, dificultad en el control de impulsos y falta de claridad emocional. Dichos resultados concuerdan con las investigaciones realizadas por Olweus (1994) y Neary y Joseph (1994) cuyos hallazgos mencionan que jóvenes víctimas muestran menor desempeño y capacidades emocionales, incluyendo la tendencia a inhibir las expresiones emocionales y el comportamiento espontáneo (Garner \& Hinton, 2010; Olweus, 1994; Perry, Kusel, \& Perry, 1988). En ese sentido, es evidente que las víctimas muestran limitaciones en el desempeño emocional, dichas limitaciones son comprensibles si se toma en cuenta que presumiblemente el objetivo es evitar futuras amenazas o ataques (Mallmann, Lisboa, \& Zanatta 2018).

$\mathrm{El}$ análisis de regresión permitió concluir que para la presente muestra que el modelo para el factor abusador y explicó $23 \%$ de la variancia. Las variables mantenidas en el modelo fueron sexo, grado de instrucción de la madre, padres viviendo juntos, extroversión, socialización y el estilo parental autoritativo (negativamente asociado). Mientras tanto para el factor víctima, el modelo explicó 18\% de la varianza e incluyó las variables sexo, edad, abertura, neuroticismo, realización, limitación de estrategias emocionales y poca claridad en objetivos planteados. Dicho hallazgo es significativo considerando que diversos estudios resaltan la importancia de factores que van más allá del contexto escolar y en cambio se vinculan también con factores familiares, personales y ambientales dentro los que se puede incluir los estilos parentales, la personalidad y la regulación emocional. (Valdés, Martínez, \& Torres, 2012). Tomando en cuenta la relevancia y las múltiples causas del fenómeno bullying, el presente estudio buscó expandir el análisis analizando variables poco analizadas en conjunto, por esa razón los resultados aquí mostrados podrían ser tomados en cuenta al momento de planificar e implementar estrategias preventivas o correctivas.

Los hallazgos en el presente estudio demuestran que, aunque el fenómeno bullying ha captado la atención de investigadores y diversos actores de la sociedad en las décadas recientes, aún se observan diversas lagunas que impiden comprender de manera integral el fenómeno. Dicha comprensión podría traer respuestas que expliquen no sólo las causas y consecuencias de este, sino también permitiría identificar anticipadamente potenciales riesgos y con ello, implementar estrategias de prevención que posibiliten mitigar sus efectos.

Por otro lado, respecto de las limitaciones del presente estudio es relevante destacar que los participantes de la investigación fueron alumnos de escuelas estatales que respondieron a los test en sala de aula de manera simultánea. Lo anterior produce un riesgo de sesgo en las respuestas, considerando que la influencia del contexto podría incentivar respuestas socialmente aceptables. Paralelamente, el hecho de ser un estudio transversal impide que las inferencias respecto del mismo sean mantenidas en el tiempo, por lo que se considera pertinente promover estudios longitudinales que evalúen el fenómeno y puedan ofrecer una comprensión más amplia de este. Además, el hecho de usar escalas de autorelato es otra potencial limitación tomando en cuenta que los participantes tienen la opción de responder lo que es socialmente correcto y no lo que realmente hacen o piensan (Aiken, 2003).

Finalmente, cabe suponer que ligado al fenómeno bullying convergen diversas variables no analizadas en el presente estudio. Sin embargo, consideramos que el presente estudio contribuye en identificar y abrir nuevas rutas de investigación para un fenómeno vigente y que por su relevancia debe ser abordado desde múltiples perspectivas. 


\section{Referencias}

Ahmed, E., \& Braithwaite, V. (2004). Bullying and victimization: Cause for concern for both families and schools. Social Psychology of Education, 7, 35-54.

Aiken, L. R. (2003). Tests psicológicos y evaluación. México, Pearson Educación.

Andrés, M., Castañeiras, C., Canet Juric, L., \& Richaud de Minzi, M. (2016). Relaciones de la regulación emocional y la personalidad con la ansiedad y depresión en niños. Avances En Psicología Latinoamericana, 34(1), 99-115. doi: http://dx.doi.org/10.12804/apl34.1.2016.07

Atik G. \& Güneri O. (2013). Bullying and victimization: Predictive role of individual, parental and academic factors School Psychology International, 34, 658-673.

Baldry, A. C., \& Farrington, D. P. (2000). Bullies and delinquents: Personal characteristics and parental styles. Journal of Community \& Applied Social Psychology, 10(1), 17-31. http://onlinelibrary.wiley.com/doi/10.1002/(SICI)1099-1298(200001/02)10:1\%3C17::AIDCASP526\%3E3.0.CO;2-M/abstract

Baldry, A. C., \& Farrington, D. P. (2004). Evaluation of an intervention program for the reduction of bullying and victimization in schools. Aggressive Behavior, 30(1), 1-15.

Balakrishnan, V., Khan, S., Fernandez, T., \& Arabnia, H. R. (2019). Cyberbullying detection on twitter using Big Five and Dark Triad features. Personality and Individual Differences, 141, 252-257.

Berger, K.S. (2007). Update on bullying at school: Science forgotten? Developmental Review, 27, 90-126.

Binsfeld, A. \& Lisboa, C. (2010). Bullying: um estudo sobre papeis sociais, ansiedade e depressão no contexto escolar do Sul do Brasil. Interpersona: an International Journal on Personal Relationships, 4, 74-105.

Boulton, M. J. \& Smith, P. K. (1994). Bully/victim problems in middle-school children: Stability, self-perceived competence, peer perceptions, and peer acceptance. British Journal of Developmental Psychology, 12, 315-329.

Baumrind, D. (1967). Child care practices anteceding three patterns o preschool behavior. Genetic Psychology Monographs, 75, 43-88

Byrne, B.J. (1994). Bullies and victims in a school setting with reference to some Dublin schools. The Irish Journal of Psychology, 15, 574-586.

Caballo, V.E., Caldereron, M., Arias, B., Salazar, I.C, \& Irurtia, M. (2012). Desarrollo y Validación de una medida de autoifnorme para evaluar el acoso escola (bullying). Behavioral Psychology/Psicologia Conductual, Vol. 20, (3), 625-647. https://www.researchgate.net/profile/Vicente_Caballo/publication/259481844_Development_ and_validation_of_a_new_selfreport_assessment_measure_of_bullying_Desarrollo_y_validac ion_de_una_nueva_medida_de_autoinforme_para_evaluar_el_acoso_escolar_bullying/links/0 deec52c1c440ee128000000.pdf

Cavalcanti, J. G., \& Pimentel, C. E. (2016). Personality and aggression: A contribution of the General Aggression Model. Estudos de Psicologia (Campinas), 33(3), 443-451.

Caravita, S., Di Blasio, P., \& Salmivalli, C. (2009). Unique and interactive effects of empathy and s ocial status on involvement in bullying. Social development, 18(1), 140-163.

Caravita, S. C., Di Blasio, P., \& Salmivalli, C. (2010). Early adolescents' participation in bullying: Is ToM involved?. The Journal of Early Adolescence, 30(1), 138-170.

Connolly I. \& O’Moore M. (2003). Personality and family relations of children who bully. Personality and Individual Differences, 35, 559- 567.

Costa, F., Teixeira, M. A., \& Gomes, W. (2000). Responsividade e exigência: duas escalas para avaliar estilos parentais. Psicologia: Reflexão e Crítica, 13, 465-473. http://www.scielo.br/pdf/prc/v13n3/v13n3a14.pdf

Costa, P. T. \& McRae, R. R. (1992). Revised NEO Personality Inventory (TM) and NEO Five-Factor Inventory (NEO-FFI) professional manual. Odessa, FL: Psychological Assessment Resources.

Coutinho, J., Ribeiro, E., Ferreirinha, R. \& Dias, P. (2009). Versão portuguesa da Escala de 
Dificuldades de Regulação Emocional e sua relação com sintomas psicopatológicos. Revista de Psicologia Clinica, 37, 145-151.

Craig, W. M. \& Pepler, D. J. (1997). Observations of bullying and victimization in the school yard. Canadian Journal of School Psychology, 13, 41-59.

Cuervo, A. A. V., Murrieta, M. U., \& Martínez, E. C. A. (2018). Disciplina restaurativa, apoyo escolar, autoeficacia, habilidades sociales y victimización por pares. Revista Interamericana de Psicología, 52(1).

Darling, N., \& Steinberg, L. (1993). Parenting style as context: An integrative model. Psychological bulletin, 113(3), 487.

Dell'Aglio, D. D., \& Hutz, C. S. (2004). Depressão e desempenho escolar em crianças e adolescentes institucionalizados. Psicologia: Reflexão e Crítica, 17(3), 351-357. http://www.scielo.br/pdf/prc/v17n3/a08v17n3.pdf

Domingues, A. E., Natividade, J., \& Hutz,S., (2011). Uso de Drogas e Estilos Parentais Percebidos na Adolescência Gerais: Revista Interinstitucional de Psicologia, 4, 3-11.

Elgar, F. J., Pickett, K. E., Pickett, W., Craig, W., Molcho, M., Hurrelmann, K., \& Lenzi, M. (2013). School bullying, homicide and income inequality: a cross-national pooled time series analysis. International journal of public health, 1-9. https://www.ncbi.nlm.nih.gov/pubmed/22714137

Espelage, D. L., Rose, C. A., \& Polanin, J. R. (2015). Social-emotional learning program to reduce bullying, fighting, and victimization among middle school students with disabilities. Remedial and special education, 36(5), 299-311.

Frizzo, M., Bisol, L. \& Lara, D. (2013). Bullying victimization is associated with dysfunctional emotional traits and affective temperaments. Journal of Affective Disorders, 148, 48-52.

Garnefski, N. \& Okma, S. (1996). Addiction-risk and aggression/criminal behavior in adolescence: Influence of family, school and peers. Journal of Adolescence, 19, 501-512.

Garner, P. W., \& Lemerise, E. A. (2007). The roles of behavioral adjustment and conceptions of peers and emotions in preschool children's peer victimization. Development and Psychopathology, 19(01), 57-71.

Garner, P. W., \& Hinton, T. S. (2010). Emotional display rules and emotion self-regulation: Associations with bullying and victimization in community-based after school programs. Journal of Community \& Applied Social Psychology, 20(6), 480-496. http://onlinelibrary.wiley.com/doi/10.1002/casp.1057/abstract

Glaso, L., Matthiesen S. B., Nielsen, M. B. \& Einarsen, S. (2007). Do targets of workplace bullying portray a general victim personality profile? Scandinavian Journal of Psychology, 48: 313-319.

Georgiou, S. N. (2008). Parental style and child bullying and victimization experiences at school.

Social Psychology of Education: An International Journal, 11(3), 213-227. https://link.springer.com/article/10.1007\%2Fs11218-007-9048-5?LI=true

Georgiou, S. N., \& Fanti, K. A. (2010). A transactional model of bullying and victimization.
Social
Psychology
of
Education,
$13(3)$,
295-311. https://link.springer.com/article/10.1007/s11218-010-9116-0

Gómez-Ortiz, O., Del Rey, R., Romera, E., \& Ortega-Ruiz, R. (2015). Los estilos educativos paternos y maternos en la adolescencia y su relación con la resiliencia, el apego y la implicación en acoso escolar. Anales De Psicología,31(3), 979-989. doi:10.6018/analesps.31.3.180791 http://www.redalyc.org/pdf/167/16741429024.pdf

Gosling, S. D., Rentfrow, P. J., \& Swann Jr, W. B. (2003). A very brief measure of the Big-Five personality domains. Journal of Research in personality, 37(6), 504-528.

Gratz, K. L., \& Roemer, L. (2004). Multidimensional assessment of emotion regulation and dysregulation: Development, factor structure, and initial validation of the difficulties in emotion regulation scale. Journal of Psychopathology and Behavioral Assessment, 26(1), 41-54. https://link.springer.com/article/10.1023/B:JOBA.0000007455.08539.94

Jiménez-Barbero, J. A., Ruiz-Hernández, J. A., Velandrino-Nicolás, A. P., \& Llor-Zaragoza, L. (2016). Actitudes hacia la violencia, impulsividad, estilos parentales y conducta externalizada en adolescentes: comparación entre una muestra de población general y una muestra clínica. anales de psicología, 32(1), 132-138. 
John, O.P. \& Srivastava, S. (1999) 'The Big Five Trait Taxonomy: History, Measurement, and Theoretical Perspectives', in L.A. Pervin and O.P. John (eds) Handbook of Personality: Theory and Research, 2nd edn, pp. 102-38. New York: Guilford. http://moityca.com.br/pdfs/bigfive_John.pdf

Kochenderfer-Ladd, B. (2004). Peer victimization: the role of emotions in adaptative and maladaptative coping. Social Development, 13, 3, 329-249.

Ladd, G. W. (1992). Themes and theories: Perspectives on processes in family peer relationships. In R. D. Park, \& G. W. Ladd (Eds.), Family-peer relationships:Models of linkage (pp. 3-34). Hillsdale, NJ: Lawrence Erlbaum Associates.

Leff, S. S., Power, T. J., Costigan, T. E., \& Manz, P. H. (2003). Assessing the Climate of the Playground and Lunchroom: Implications for Bullying Prevention Programming. School Psychology Review.

Lereya, S. T., Samara, M., \& Wolke, D. (2013). Parenting behavior and the risk of becominga victim and a bully/victim: A meta-analysis study. Child abuse \& neglect.

López N. A. \& Saavedra, L. (2003). Diga Não ao Bullying: programa de redução do comportamento agressivo entre estudantes - Resultados parciais. Recuperado em 01 out. 2014 de http://www.observatoriodainfancia.com.br.

Lynn Hawkins, D., Pepler, D. J. \& Craig, W. M. (2001), Naturalistic Observations of Peer Interventions in Bullying. Social Development, 10: 512-527. http://onlinelibrary.wiley.com/doi/10.1111/1467-9507.00178/full

Maccoby, E. E. y Martin, J. A. (1983). Socialization in the context of the family. Parent-child interaction. En P. Mussen (Ed.), Handbook of child psychology (vol 4: Socialization, personality and social development). pp. 1-101. New York: John Wiley y Sons.

Mahady Wilton, M. M., Craig, W. M., \& Pepler, D. J. (2000). Emotional regulation and display in classroom victims of bullying: Characteristic expressions of affect, coping styles and relevant contextual factors. Social Development, 9(2), 226-245. http://onlinelibrary.wiley.com/doi/10.1111/1467-9507.00121/abstract

Mallmann, C. L., de Macedo Lisboa, C. S., \& Zanatta Calza, T. (2018). Cyberbullying and coping strategies in adolescents from Southern Brazil. Acta Colombiana de Psicología, 21(1), 13-43.

Millan Reyes, L. C.; Barrera Sanchez, L. F. \& Ospina Diaz, J. M. (2015). Caracterización del bullying en estudiantes de medicina de Tunja, Boyacá. Revista Virtual Universidad Católica del Norte, 45, 101-112. http://revistavirtual.ucn.edu.co/index.php/RevistaUCN/article/viewFile/659/1191

Mynard, H., \& Joseph, S. (1997). Bully/victim problems and their association with Eysenck's personality dimensions in 8 to 13 year-olds. British Journal of Educational Psychology, 67, 5154.

Neary, A., \& Joseph, S. (1994). Peer victimization and its relationship to self-concept and depression among schoolgirls. Personality and Individual Differences, 16(1), 183-186.

Nunes, C., Hutz, C. \& Nunes M. (2010). Bateria Fatorial de Personalidade. Manual Técnico. Casa do Psicólogo. São Paulo.

Ok, S. \& Aslan, S. (2010). The school bullying and perceived parental style in adolescents. Procedia Social and Behavioral Sciences, 5, 536-540.

Olweus, D. (1993). Bullying at school: What we know and what we can do. Oxford: MA: Blackwell Publishers.

Olweus, D. (1994). Bullying at school: basic facts and effects of a school based intervention program. Journal of child psychology and psychiatry, 35(7), 1171-1190.

Olweus, D. (2006). Bullying at school: What we know and what we can do. Cambridge, MA: Blackwell Publishers, Inc. (Original work published 1993).

Olweus, D. (2011). Bullying at school and later criminality: Findings from three Swedish community samples of males. Criminal behaviour and mental health, 21(2), 151-156.

O’Connell, P., Pepler, D. \& Craig, W. (1999) Peer involvement in bullying: insights and challenges for intervention. Journal of Adolescence, 22, 437-452.

Perry, D., Kusel, S., \& Perry, L. (1988). Victims of peer aggression. Child Development, 24, 807-814. 
Pontzer, D. (2010). A theoretical test of bullying behavior: Parenting, personality and the bully/victim relationship. Journal of Family Violence, 25(3), 259-273.

Salmivalli, C., Huttunen, A. \& Lagerspetz, K. (1997). Peer networks and bullying in schools. Scandinavian Journal of Psychology, 38, 305-312. http://onlinelibrary.wiley.com/doi/10.1111/1467-9450.00040/full

Sanchez, V., Ortega, R. \& Menesini, E. (2012). La competencia emocional de agresores y victimas de bullying. Anales de Psicologia, 28, 71-82.

Sansone, R., Lamc, C. \& Wiederm M. (2010). Bullied in childhood: correlations with borderline personality in adulthood. Comprehensive Psychiatry, 51, 458-461. http://linkinghub.elsevier.com/retrieve/pii/S0010440X10000052?via=sd

Sansone, R. A., \& Sansone, L. A. (2008). Bully victims: Psychological and somatic aftermaths. Psychiatry, 6, 62-64.

Seals D, \& Young J. (2003). Bullying and victimization: prevalence and relationship to gender, grade level, ethnicity, self-esteem, and depression. Adolescence, 38, 735-747.

Silva, J., Oliveira, W., Mello, F., Prado, R., Silva, M., \& Malta, D. (2019). Prevalência da prática de bullying referida por estudantes brasileiros: dados da Pesquisa Nacional de Saúde do Escolar, 2015. Epidemiologia e Serviços de Saúde, 28(2).

Smetana, J. G. (2017). Current research on parenting styles, dimensions, and beliefs. Current opinion in psychology, 15, 19-25.

Springer, A. E., Cuevas Jaramillo, M. C., Ortiz Gómez, Y., Case, K., \& Wilkinson, A. (2016). School social cohesion, student-school connectedness, and bullying in Colombian adolescents. Global health promotion, 23(4), 37-48.

SPSS Inc. Released 2008. SPSS Statistics for Windows, Version 17.0. Chicago: SPSS Inc.

Sutton, J. \& Keogh, E. (2000). Social Competition in School: Relationships with Bullying, Machiavellianism, and Personality, British Journal of Educational Psychology 70, 443-56.

Tani, F., Greenman, P., Schneider, B. \& Fregoso, M. (2003). Bullying and the Big Five: A Study of Childhood Personality and Participant Roles in Bullying Incidents. School Psychology International, 24, (2) 131-146.

Valdés, A., Martínez, C. \& Torres G. (2012). Diferencias en la situación socioeconómica, clima y ajuste familiar de estudiantes con reportes de bullying y sin ellos. Psicología desde el Caribe, 29(3), 616-631. Retrieved March 31, 2017, from http://www.scielo.org.co/scielo.php?script=sci_arttext\&pid=S0123417X2012000300004\&lng=en\&tlng=.

Valle, M. D., Betegón, E., \& Irurtia, M. J. (2018). Efecto del uso de estrategias cognitivas de regulación emocional sobre la ansiedad en adolescentes españoles. Suma Psicológica, 25(2), 153-161.

Wang, J., Iannotti, R. J., \& Nansel, T. R. (2009). School bullying among adolescents in the United States: physical, verbal, relational, and cyber. The Journal of Adolescent Health, 45, 368-375.

WHO. (2001). The world health report - Mental Health: New Understanding, New Hope. World Health Organization. Geneva. from http://www.who.int/whr/2001/en/.

WHO. WHO Press. (2005). Atlas. Child and adolescent mental health resources: global concerns, implications for the future. Department of Mental Health and Substance Abuse. Geneva.

Wilton, M., \& Craig, W. (2000). Emotional Regulation and Display in Classroom Victims of Bullying: Characteristics Expressions of affect, Coping styles and Relevant Contextual Factors. Social Development, 9 (2) 226-245.

Winsper, C., Lereya, T., Zanarini, M., \& Wolke, D. (2012). Involvement in bullying and suiciderelated behavior at 11 years: A Prospective birth cohort study. Journal of the American Academy of Child and Adolescent Psychiatry, 51, 271-282.

Wittig, S. M., \& Rodriguez, C. M. (2019). Interaction between maternal and paternal parenting styles with infant temperament in emerging behavior problems. Infant Behavior and Development, 57, 101323.

Wolke, D., \& Samara, M. (2004). Bullied by siblings: Association with peer victimization and 
behavior problems in Israeli lower secondary schoolchildren. Journal of Child Psychology and Psychiatry, 45, 1015-1029. http://onlinelibrary.wiley.com/doi/10.1111/j.1469-7610.2004.t011-00293.x/abstract

Zhang, A., De Luca, S., Oh, S., Liu, C., \& Song, X. (2019). The moderating effect of gender on the relationship between bullying victimization and adolescents' self-rated health: An exploratory study using the Fragile Families and Wellbeing Study. Children and Youth Services Review, 96, 155-162.

Zhang, A., Liu, C., Bornheimer, L. A., Solomon, P., Wang, K., \& Morrow, S. P. (2019). The indirect effect of bullying on adolescent self-rated health through mental health: A gender specific pattern. Children and Youth Services Review, 10, 43-85. 